\title{
Composing Trust Models towards Interoperable Trust Management ${ }^{\star}$
}

\author{
Rachid Saadi, Mohammad Ashiqur Rahaman, \\ Valérie Issarny, and Alessandra Toninelli \\ ARLES Project-Team \\ INRIA CRI Paris-Rocquencourt, France \\ \{name.surname\}@inria.fr
}

\begin{abstract}
Computational trust is a central paradigm in today's Internet as our modern society is increasingly relying upon online transactions and social networks. This is indeed leading to the introduction of various trust management systems and associated trust models, which are customized according to their target applications. However, the heterogeneity of trust models prevents exploiting the trust knowledge acquired in one context in another context although this would be beneficial for the digital, ever-connected environment. This is such an issue that this paper addresses by introducing an approach to achieve interoperability between heterogeneous trust management systems. Specifically, we define a trust meta-model that allows the rigorous specification of trust models as well as their composition. The resulting composite trust models enable heterogeneous trust management systems to interoperate transparently through mediators.
\end{abstract}

\section{Introduction}

With people getting increasingly connected virtually, trust management is becoming a central element of today's open distributed digital environment. However, existing trust management systems are customized according to specific application domains, hence implementing different trust models. As a result, it is nearly impossible to exploit established trust relations across systems. While a trust relation holding in one system does not systematically translate into a similar relation in another system, it is still a valuable knowledge, especially if the systems relate to the same application domains (e.g., e-commerce, social network). This is such an issue that we are addressing in this paper.

To the best of our knowledge, little work investigates interoperability between heterogeneous trust models. The closest to our concern is the work of [19], which describes a trust management architecture that enables dealing with a variety of trust metrics and mapping between them. However, the architecture deals with the composition at the level of trust values and do not account for the variety of trust models. In particular, one may want to differentiate between direct trust values and reputation-based ones when composing them. In general, what is needed is a way to formalize heterogeneous trust models and their composition. Such a concern is in particular addressed in [9]21], which introduce trust meta-models based on state of the art trust management systems. Nevertheless, little detail is given and the paper does not describe how

\footnotetext{
* Work supported by EU-funded project FP7-231167 CONNECT and by EU-funded project FP7-256980 NESSOS.
} 
to exploit the meta-model for composing heterogeneous trust models and this achieve interoperability. Dealing with the heterogeneity of trust models is also investigated in [420]. However, the study is for the sake of comparison and further concentrates on reputation-based models. Summarizing, while the literature is increasingly rich of trust models, dealing with their composition remains a challenge.

Towards overcoming the interoperability challenge faced by trust management systems, this paper introduces a comprehensive approach based on the definition of a reference trust meta-model. Specifically, based on the state of the art (Section 2), the trust meta-model formalizes the core entities of trust management systems, i.e., trust roles, metrics, relations and operations (Section 3). The trust meta-model then serves specifying the composition of trust models in terms of mapping rules between roles, from which trust mediators are synthesized (Section 4). Trust mediators transparently implement mapping between respective trust relations and operations of the composed models. While this paper introduces the composition approach from a theoretical perspective, we are currently implementing it as part of the CONNECT project 1 on next generation middleware for interoperability in complex systems of systems (Section 5).

\section{Trust Model Definition}

As in particular defined in [5]: i.e., A trustor trusts a trustee with regard to its ability to perform a specific action or to provide a specific service. Hence, any trust model may basically be defined in terms of the three following elements:

1. Trust roles abstract the representative behaviors of stakeholders from the standpoint of trust management, in a way similar to role-based access control model [3].

2. Trust relations serve specifying trust relationships holding among stakeholders, and

3. Trust assessment define how to compute the trustworthiness of stakeholders.

We further define trust relations and assessment below.

\subsection{Trust Relations}

We identify two types of trust relationships, i.e., direct and indirect, depending on the number of stakeholders that are involved to build the trust relationship:

Direct trust: A direct trust relationship represents a trust assertion of a subject (i.e., trustor) about another subject (i.e., trustee). It is thus a one-to-one trust relation (denoted 1:1)) since it defines a direct link from a trustor (1) to a trustee (1). One-to-one trust relations are maintained locally by trustors and represent the trustors' personal opinion regarding their trustees [10]. For example, a one-to-one relation may represent a belonging relationship (e.g., employees trust their company), a social relationship (e.g., trust among friends), or a profit-driven relationship (e.g., a person trusts a trader for managing its portfolio).

Recommendation-based trust: As opposed to a direct trust relationship, a recommendation-based relationship represents a subject's trustworthiness based on a third party's opinion. This can be either (i) transitive-based or (ii) reputation-based.

\footnotetext{
${ }^{1} \mathrm{http}: / /$ connect-forever.eu/
} 
Transitive-based trust relations are one-to-many (denoted 1:N). Such a relation enables a trustor (1) to indirectly assess the trustworthiness of an unknown trustee through the recommendations of a group of trustees $(\mathbf{N})$. Hence, the computation of $1: \mathrm{N}$ relations results from the concatenation and/or aggregation of many 1:1 trust relations. The concatenation of 1:1 trust relations usually represents a transitive trust path, where each entity can trust unknown entities based on the recommendation of its trustees. Thus, this relationship is built by composing personal trust relations [118]. Furthermore, in the case where there exist several trust paths that link the trustor to the recommended trustee, the aggregation can be used to aggregate all given trust recommendations [7].

Reputation-based trust relations are many-to-one (denoted $N: 1$ ) and result from the aggregation of many personal trust relationships having the same trustee. Hence, the $\mathrm{N}$ :1 trust relation allows the definition of the reputation of each trustee within the system. Reputation systems may then be divided into two categories depending on whether they are (i) Centralized or (ii) Distributed. With the former, the reputation of each participant is collected and made publicly available at a centralized server (e.g., eBay, Amazon, Google, [14]). With the latter, reputation is spread throughout the network and each networked entity is responsible to manage the reputation of other entities (e.g., [7|23]).

\subsection{Trust Assessment}

Trust assessment, i.e., assigning values to trust relationships, relies on the definition of: (i) trust metrics characterizing how trust is measured and (ii) operations for composing trust values.

Trust metrics: Different metrics have been defined to measure trust. This is due to the fact that one trust metric may be more or less suitable to a certain context. Thus, there is no widely recognized way to assign trust values. Some systems assume only binary values. In [24], trust is quantified by qualitative labels (e.g., high trust, low trust etc.). Other solutions represent trust by a numerical range. For instance, this range can be defined by the interval [-1..1] (e.g., [12]), [0..n] (e.g., [1]18]) or [0..1] (e.g., [7]). A trust value can also be described in many dimensions, such as: (Belief, Disbelief, Uncertainty) [7].

In addition, several definitions exist about the semantics of trust metrics. This is for instance illustrated by the meaning of zero and negative values. For example, zero may indicate lack of trust (but not distrust), lack of information, or deep distrust. Negative values, if allowed, usually indicate distrust, but there is a doubt whether distrust is simply trust with a negative sign, or a phenomenon of its own.

Trust operations: We define four main operations for the computation of trust values associated with the trust relations given in Section 2.1 (see table 1): bootstrapping, refreshing, aggregation, and concatenation.

The bootstrapping operation initializes the a priori values of $1: 1$ and $\mathrm{N}: 1$ trust relations. Trust bootstrapping consists of deciding how to initialize trust relations in order to efficiently start the system and also allow newcomers to join the running system [16]. Most existing solutions simply initialize trust relation with a fixed value (e.g., 0.5 [6], a uniform Beta probabilistic distribution [8]). Other approaches include among 
Table 1. Trust assessment operations

\begin{tabular}{|l||c|c|c|c|}
\hline & Bootstrapping & Aggregation & Concatenation & Refreshing \\
\hline One-to-One $(1: 1)$ & $\mathrm{X}$ & & & $\mathrm{X}$ \\
\hline One-to-Many (1:N) & & $\mathrm{X}$ & $\mathrm{X}$ & \\
\hline Many-to-One (N:1) & $\mathrm{X}$ & $\mathrm{X}$ & & $\mathrm{X}$ \\
\hline
\end{tabular}

others: initializing existing trust relations according to given peers recommendations [17]; applying a sorting mechanism instead of assigning fixed values [18]; and assessing trustees into different contexts (e.g., fixing a car, babysitting, etc.) and then inferring unknown trust values from known ones of similar or correlate contexts [162].

All the solutions dealing with 1:N trust assessment mainly define the concatenation and the aggregation operations, in order to concatenate and to aggregate trust recommendations by computing the average [18], the minimum or the product [1] of all the intermediary trust values. In the case of Web service composition, some approaches (e.g., [15]) evaluate the recommendation for each service by evaluating its provider, whereas other approaches (e.g., [11]) evaluate the service itself in terms of its previous invocations, performance, reliability, etc. Then, trust is composed and/or aggregated according to the service composition flow (sequence, concurrent, conditional and loop).

Aggregation operations such as Bayesian probability (e.g., [13]) are often used for the assessment of N:1 (reputation-based) trust relations. Trust values are then represented by a beta Probability Density Function [8], which takes binary ratings as inputs (i.e., positive or negative) from all trustors. Thus, the reputation score is refreshed from the previous reputation score and the new rating [14]. The advantage of Bayesian systems is that they provide a theoretically sound basis for computing reputation scores and can also be used to predict future behavior.

Finally, refreshing operations are mainly trigged by trustors to refresh $1: 1$ and $\mathrm{N}: 1$ trust relations, after receiving stakeholders' feedback.

\section{Trust Meta-model}

Following the above, we formally define the trust meta-model as: $T M=<\mathbb{R}, \mathbb{L}, \mathbb{M}, \mathbb{O}>$, where $\mathbb{R}, \mathbb{L}, \mathbb{M}$ and $\mathbb{O}$ are the finite sets of trust roles, relations, metrics and operations.

\subsection{Trust Meta-model Formalization}

As detailed below, each set of $T M$ consists of elements where an element can have a simple value (e.g., string) or a complex value. A complex value of an element is either an exclusive combination of values (only one of the values) $\vee v\left(\right.$ e.g., $v_{1} \vee v_{2} \vee v_{3}$ ) or an inclusive combination of values (one or more elements) $\diamond v$ (e.g., $v_{1} \wedge v_{2} \wedge\left(v_{3} \vee v_{4}\right)$ ) of elements.

Role set $\mathbb{R}$ : The role set contains all the roles $r$ played by the stakeholders of the trust model. A role $r$ of $\mathbb{R}$ is simply denoted by its name:

$$
r=<\text { name:string }>
$$


where: the attribute name of type string represents the name or the identifier of the role2. In our meta-model, a stakeholder is represented as a Subject s, playing a number of roles, $r_{1}, r_{2} \ldots$ and $r_{n}$, which is denoted as $s \triangleright r_{1}, r_{2} \ldots r_{n}$.

Metric set $\mathbb{M}$ : The metric set describes all the trust metrics that can be manipulated by the trust model. A metric is formally denoted as a pair:

$$
m=<\text { name:string, type:string }>
$$

where: name and type are strings and respectively define the name and the type. The type can be a simple type (e.g., probability([0..1]), label(good, bad), etc.) or a composition of simples ones (e.g., tuple (believe([0..1]), uncertainty $([0 . .1]))$.

Relation set $\mathbb{L}$ : A relation set $\mathbb{L}$ contains all the trust relations that are specified by the trust model. We specifically denote a trust relation as a tuple:

$$
\begin{array}{r}
l=<\text { name:string, ctx:string, type:string, trustor: } \vee r_{i} \text {, trustee: } \vee r_{j}, \text { value: } m_{k}> \\
\text { with } r_{i}, r_{j} \in \mathbb{R} \text { and } m_{k} \in \mathbb{M}
\end{array}
$$

where: (i) name identifies the relation; (ii) ctx describes the context of the relationship in terms of the application domain (e.g., selling); (iii) type represents the cardinality of the relation and is denoted by one of the following arities: $1: 1,1: \mathrm{N}$ or $\mathrm{N}: 1$; (iv) trustor and trustee are roles where a trust relation relates a trustor role with a trustee role; (v) value is an element from the metric set and thus reflects the trust measure given by the trustor to the trustee through this relation. In the above, note that different trustors can establish the same type of relationship with different trustees. Thus, as a trust relation is binary and between a trustor role and a trustee, the exclusive combination of roles (e.g., $r_{1} \vee r_{2} \vee r_{3}$ ) is used to describe these elements

Operation set $\mathbb{O}$ : The operation set specifies the operations that can be performed over relations by a subject, either to assess the trustworthiness of another subject or to communicate (i.e., request/response) trust values associated with desired subjects (see Figure 11. As defined in Section 2, trust assessment relies on the bootstrapping, aggregation, concatenation and refreshing operations, whereas, the communication of a trust value relies on the request and response operations. An operation is formally denoted as:

$$
\begin{array}{r}
o=<\text { name:string, host: } \vee r_{i}, \text { type:string, input: } \diamond l_{j}, \text { output: } \diamond l_{k}, \text { via: } \diamond l_{n}, \text { call: } \diamond_{o}> \\
\text { Where } r_{i} \in \mathbb{R}, l_{j}, l_{k}, l_{n}, \in \mathbb{L} \text {, and } o \in \mathbb{O}
\end{array}
$$

where: (i) name identifies uniquely an operation; (ii) host instantiates the role(s) that hosts and executes the operation; (iii) type defines the operation (i.e., request, response, bootstrapping, aggregation, concatenation, and refreshing); (iv) input gives the trust

\footnotetext{
${ }^{2}$ Note that the name can in particular be specified by an ontological concept that formally describes this role into a given trust ontology although this is not longer discussed in this paper.
} 


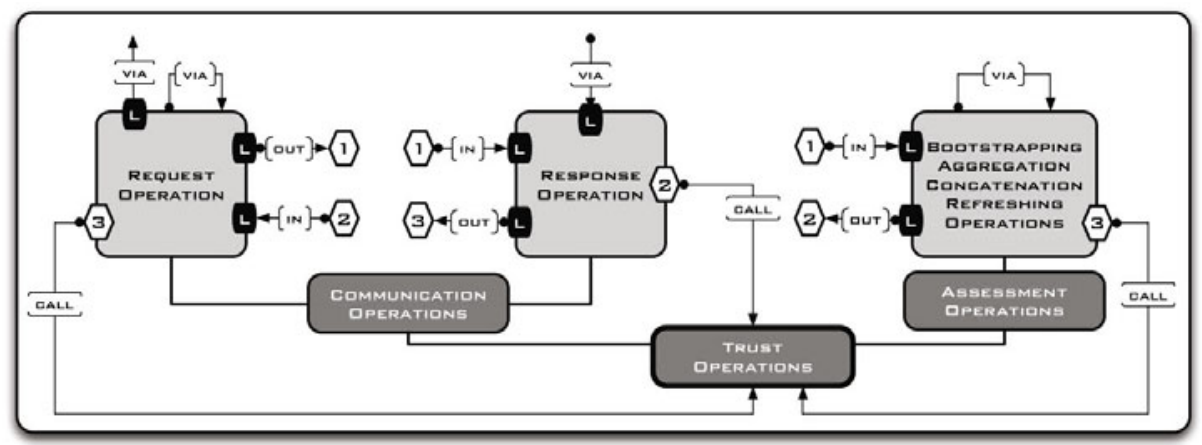

Fig. 1. Operation continuation

relations that are required to perform an assessment operation or are received by a communication operation; (v) output gives the trust relations that are provided, as the result of either an assessment operation or a communication; (vi) via specifies the trust relationship that should hold with the role with which the communication happens, while its value is self in the case of assessment; and (vii) call denotes a continuation (see Figure 1). Note that input and output are complex values, i.e., logical conjunction of one or more relations.

Trust graph TG: We associate the definition of a trust graph with any trust model $T M$ for the sake of graphical representation. Specifically, the trust graph $T G(\mathbb{R}, \mathbb{E})$ associated with a given $T M$ is a directed graph with the vertices representing the set of roles $\mathbb{R}$ of $T M$, and the set of edges $\mathbb{E}$ representing the relationship between roles according to $\mathbb{L}$. Hence, each edge is labeled by the referenced relation $l$ from the set of relations $\mathbb{L}$ and the type of that relation, i.e., $1: 1,1: \mathrm{N}$ or $\mathrm{N}: 1$.

\subsection{Example}

We illustrate the expressiveness of our trust meta-model by considering the specification of representative trust models associated with two selling transaction scenarios. Precisely, we introduce the specification of an eBay like centralized trust model (see Table 2) and of a fully distributed one (see Table 3). Both trust models aim at assessing transaction behaviors of sellers.

Figure 2 depicts the trust graphs of both models; the centralized trust model, i.e., $T M_{C}$ (on the left in the figure), is defined with three roles, i.e., $r_{S}=$ Seller, $r_{B}=$ Buyer, and $r_{M}=$ Manager, whereas the distributed trust model, i.e., $T M_{D}$ (on the right in the figure), is defined with the unique role $r_{C}=$ Customer, which can be either a seller or a buyer.

Focusing on the specification of $T M_{C}$ in Table 2, the roles Buyer and Seller have a direct trust relationship (i.e., $l_{0}$ ) with the Manager that manages the sellers' reputation (i.e., $l_{3}$ ). Thus, any Buyer can: (i) query the Manager about the reputation of a Seller (i.e., $l_{1}$ ), and (ii) provide the Manager with its feedback (i.e., $l_{2}$ ) after a selling transaction. Hence, a Buyer has to perform a request operation (i.e., $o_{4}$ ) to get the reputation of the seller, so that it can compute locally the trustworthiness of the 

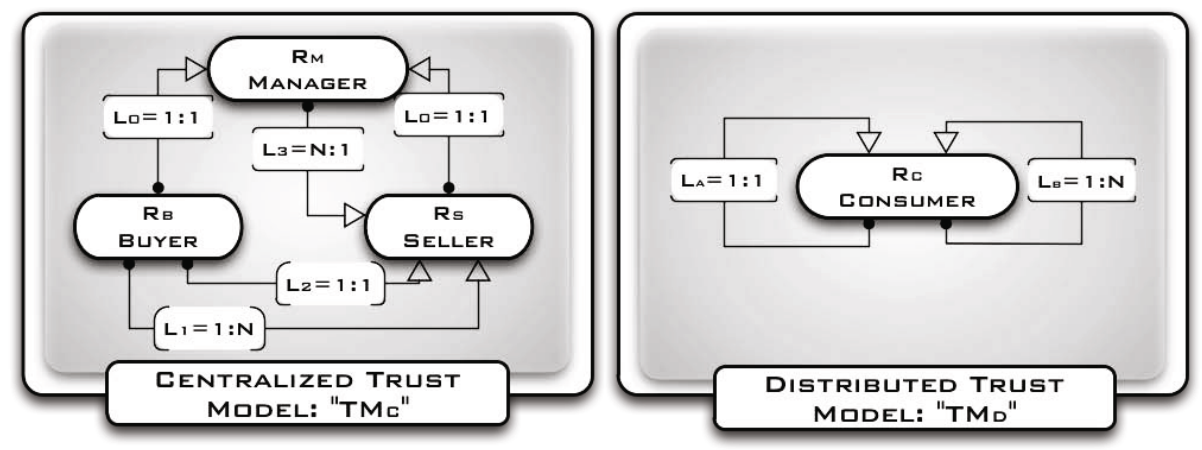

Fig. 2. Trust graphs of the centralized $\left(T M_{C}\right)$ and the distributed $\left(T M_{D}\right)$ trust models

seller (i.e., $o_{1}$ ). After a transaction is completed, a Buyer can provide its feedback to the Manager by triggering a request operation (i.e., $o_{8}$ ). The Manager in turn processes (i.e., $\left.o_{9}\right)$ this feedback request to compute and refresh the reputation of the concerned Seller (i.e., $o_{3}$ ).

Regarding the distributed model $T M_{D}$ specified in Table 3 the role Customer of the distributed model can maintain a direct trust relationship with other Customers (i.e., $l_{a}$ ) and can then ask trustee Customers to get their recommendation about unknown Customers that are sellers (i.e., $l_{b}$ ). Hence, a Customer can perform a request operation (i.e., $o_{d}$ ) to get a recommendation of an unknown Customer seller, so that the requester Customer can compute locally the trustworthiness of the Seller (i.e., $o_{b}$ and $o_{c}$ ). After the transaction is completed, the requester $C$ ustomer can provide its feedback to other Customers by triggering a request operation (i.e., $o_{f}$ ). The recipient $C$ ustomer

Table 2. Centralized Trust model: $T M_{C}$

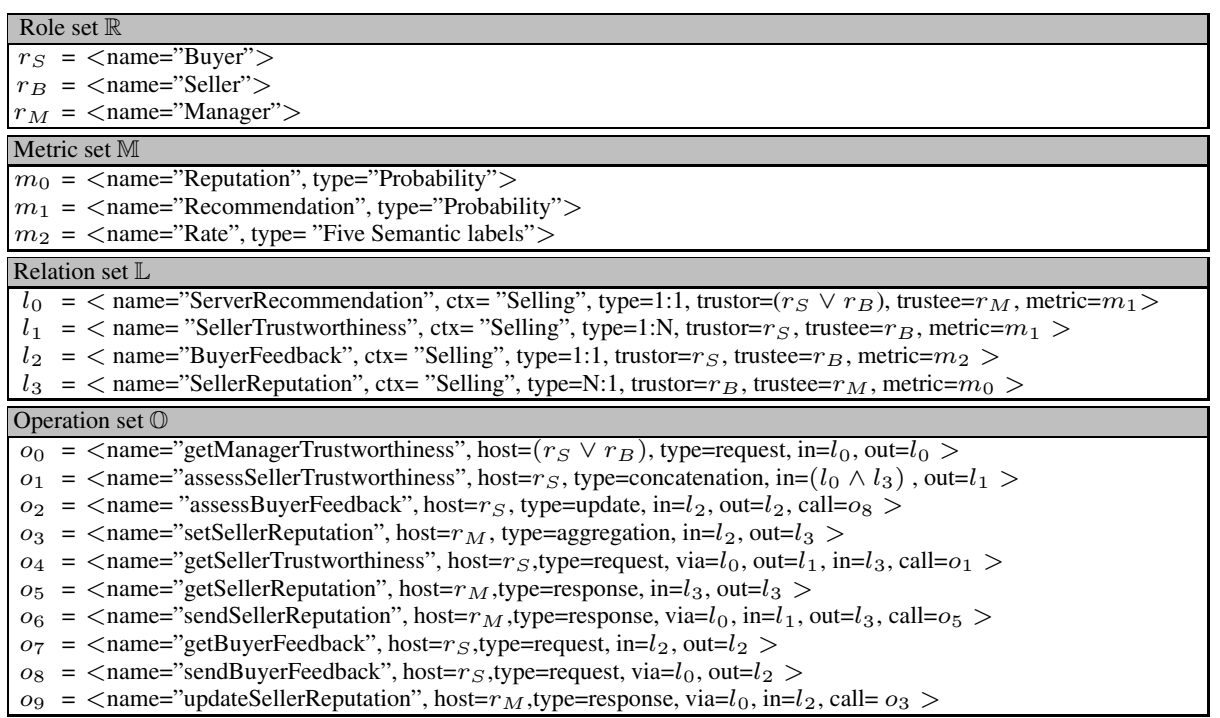


Table 3. Distributed Trust model: $T M_{D}$.

\begin{tabular}{|c|}
\hline Role set $\mathbb{R}$ \\
\hline$r_{C}=<$ name $="$ Customer " $\rangle$ \\
\hline Metric set $\mathbb{M}$ \\
\hline$m_{a}=\langle$ name="Recommendation", type="Probability" $\rangle$ \\
\hline Relation set $\mathbb{L}$ \\
\hline $\begin{array}{l}l_{a}=<\text { name="DirectCustomer Trustworthiness", ctx }=\text { "auction", type }=1: 1, \text { trustor }=r_{C}, \text { trustee }=r_{C}, \text { metric }=m_{a}> \\
l_{b}=<\text { name="TransitiveCustomer Trustworthiness", ctx="auction", type }=1: \mathrm{N}, \text { trustor }=r_{C}, \text { trustee }=r_{C}, \text { metric }=m_{a}>\end{array}$ \\
\hline Operation set $\mathbb{O}$ \\
\hline 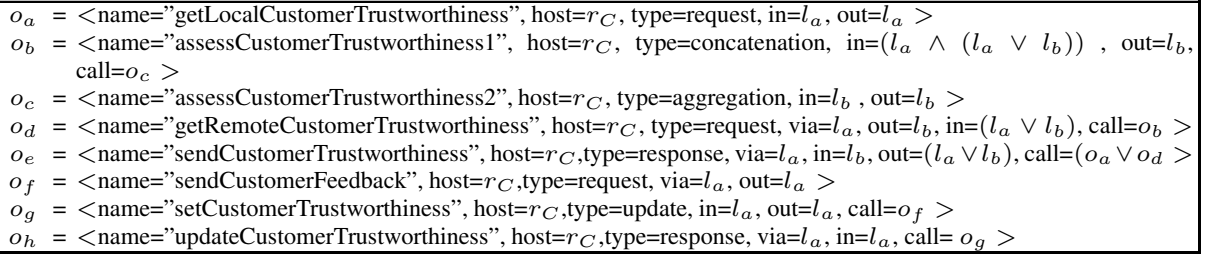 \\
\hline
\end{tabular}

can process (i.e., $o_{h}$ ) this feedback to refresh its relationship with the concerned Seller (i.e., $o_{g}$ ) and can also in turn propagate this feedback by calling the $o_{f}$.

\section{Composing Trust Models}

Given the specification of trust models, their composition relies on mapping their respective roles so that: (i) the trustworthiness of the various roles can be assessed, (ii) existing trust relations can be queried, and (iii) trust feedbacks can be propagated transparently from one trust model to another. Further, the existing trust relations and operations are extended to relate roles from the composed models, and new assessment operations are required to map trust relations from one model to another. Finally, the resulting mapping and extensions are implemented through mediation [22] so as to make composition transparent to existing systems, which leads us to introduce the corresponding mediator role.

Formally, the composition, denoted $\bigoplus$, of two trust models $T M_{x}$ and $T M_{y}$, which introduces the trust model $T M_{x y}$, is defined as follows:

$$
\begin{aligned}
T M_{x y}= & T M_{x} \bigoplus_{\Psi^{x y}} T M_{y} \\
= & <\mathbb{R}_{x}, \mathbb{M}_{x}, \mathbb{L}_{x}, \mathbb{O}_{x}>\bigoplus_{\Psi^{x y}}<\mathbb{R}_{y}, \mathbb{M}_{y}, \mathbb{L}_{y}, \mathbb{O}_{y}> \\
= & \left.\begin{array}{l}
\mathbb{R}_{x y}=\mathbb{R}_{x} \cup \mathbb{R}_{y} \cup \mu \mathbb{R}_{x y} \\
\mathbb{M}_{x y}=\mathbb{M}_{x} \cup \mathbb{M}_{y} \\
\mathbb{L}_{x y}=\mathbb{L}_{x}^{+} \cup \mathbb{L}_{y}^{+} \\
\mathbb{O}_{x y}=\mathbb{O}_{x}^{+} \cup \mathbb{O}_{y}^{+} \cup \mu \mathbb{O}_{x y}
\end{array}\right\rangle
\end{aligned}
$$

where:

- $\Psi^{x y}$ is the set of mapping rules over roles that enables the composition of $T M_{x}$ and $T M_{y}$;

- $\mu \mathbb{R}_{x y}$ and $\mu \mathbb{O}_{x y}$ are the new sets of mediator roles and mediation operations, respectively; 
- $\left(\mathbb{L}_{x}^{+}\right.$and $\left.\mathbb{L}_{y}^{+}\right)$and $\left(\mathbb{O}_{x}^{+}\right.$and $\left.\mathbb{O}_{y}^{+}\right)$are the extended relations and operations, respectively.

In the following, we elaborate on the mediation process to generate the sets of mediator roles, and mediation operations (i.e., $\mu \mathbb{R}_{x y}$, and $\mu \mathbb{O}_{x y}$ ) and extended relations and operations (i.e., $\mathbb{L}_{x}^{+}, \mathbb{O}_{x}^{+} \mathbb{L}_{y}^{+}, \mathbb{O}_{y}^{+}$).

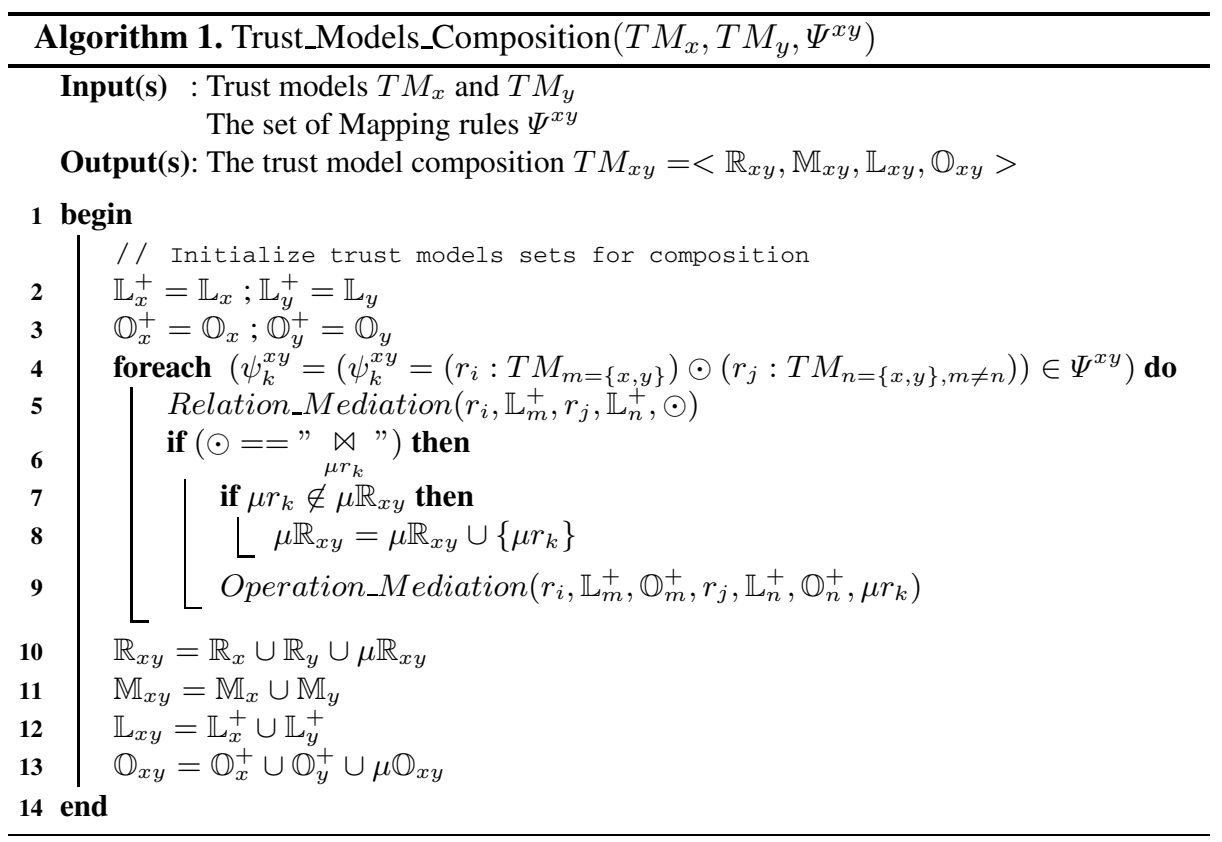

\subsection{Role Mapping}

The mapping of roles from 2 distinct models is explicitly defined through a set of mapping rules defined as follows:

$$
\psi_{k}^{s t}=\left(r_{s}: T M_{s}\right) \odot\left(r_{d}: T M_{t}\right)
$$

where, $\odot$ is asymmetric and maps the source role $r_{s}$ of $T M_{s}$ to the target role $r_{t}$ of $T M_{t}$. We further refine $\odot$ into two mapping operators:

- The See operator, noted " $\succ "$, simply associates a source role with a target role so as to define that the role $r_{t}$ of $T M_{t}$ is seen as $r_{s}$ in $T M_{t}$. For instance, in the selling transaction scenarios, $\left(r_{B}: T M_{C}\right) \succ\left(r_{C}: T M_{D}\right)$ means that Buyers (i.e., $r_{B}: T M_{C}$ ) of the centralized trust model are seen by the distributed trust model $\left(T M_{D}\right)$ as Customers $\left(r_{C}: T M_{D}\right)$.

- The Mimic operator, noted "® $\aleph_{\mu r}$ ", specifies that $r_{s}$ should be able to request trust values of $T M_{t}$ as if it was $r_{t}$. This is practically achieved through the mediator role $\mu r$ that translates $r_{s}$ requests into $r_{t}$ requests. For instance, the rule $\left(r_{C}: T M_{D}\right) \underset{\mu r}{\bowtie}$ 
$\left(r_{S}: T M_{A}\right)$ means that any customer is able to request trust values as if it was a buyer in the centralized trust management system, thanks to the mediation achieved by $\mu r$.

The computation of the composition of trust models $T M_{x}$ and $T M_{y}$ is detailed in Algorithm 1. The algorithm iterates on mapping rules for each of which it invokes Relation_Mediation (see line 5) so as to extend relation sets, namely: $\mathbb{L}_{x}^{+}$and $\mathbb{L}_{y}^{+}$, (see Section 4.2). Then, according to the definition of Mimic rules, mediator roles (i.e., $\mu r$ ) are added to the set of mediator roles (see lines 7-8), and Operation_Mediation is invoked so as to perform mediation over the communication operations (see line 9) of the composed trust models (see Section 4.3).

\subsection{Relation Mediation}

The aim of relation mediation is to extend the trust relations of the original models to roles of the other. More precisely, for any trust relation:

$l=<$ name:string, ctx:string, type:string, trustor: $\vee r_{i}$, trustee: $\vee r_{j}$, metric: $m_{k}>$ of $\mathbb{L}_{x}$ and $\mathbb{L}_{y}$ of the composed models $T M_{x}$ and $T M_{y}$, its trustee and trustor elements are possibly extended to account for mapping between roles.

Algorithm 2 details the corresponding extension where: (i) function $e \sqsupset v$ returns true if $v$ is in $e$, and (ii) $e \stackrel{v_{i}}{\longleftarrow} v_{j}$ replaces the value $v_{i}$ in $e$ with the value $v_{j}$. As shown in the algorithm, the extension of trust relations depends on the type of the mapping

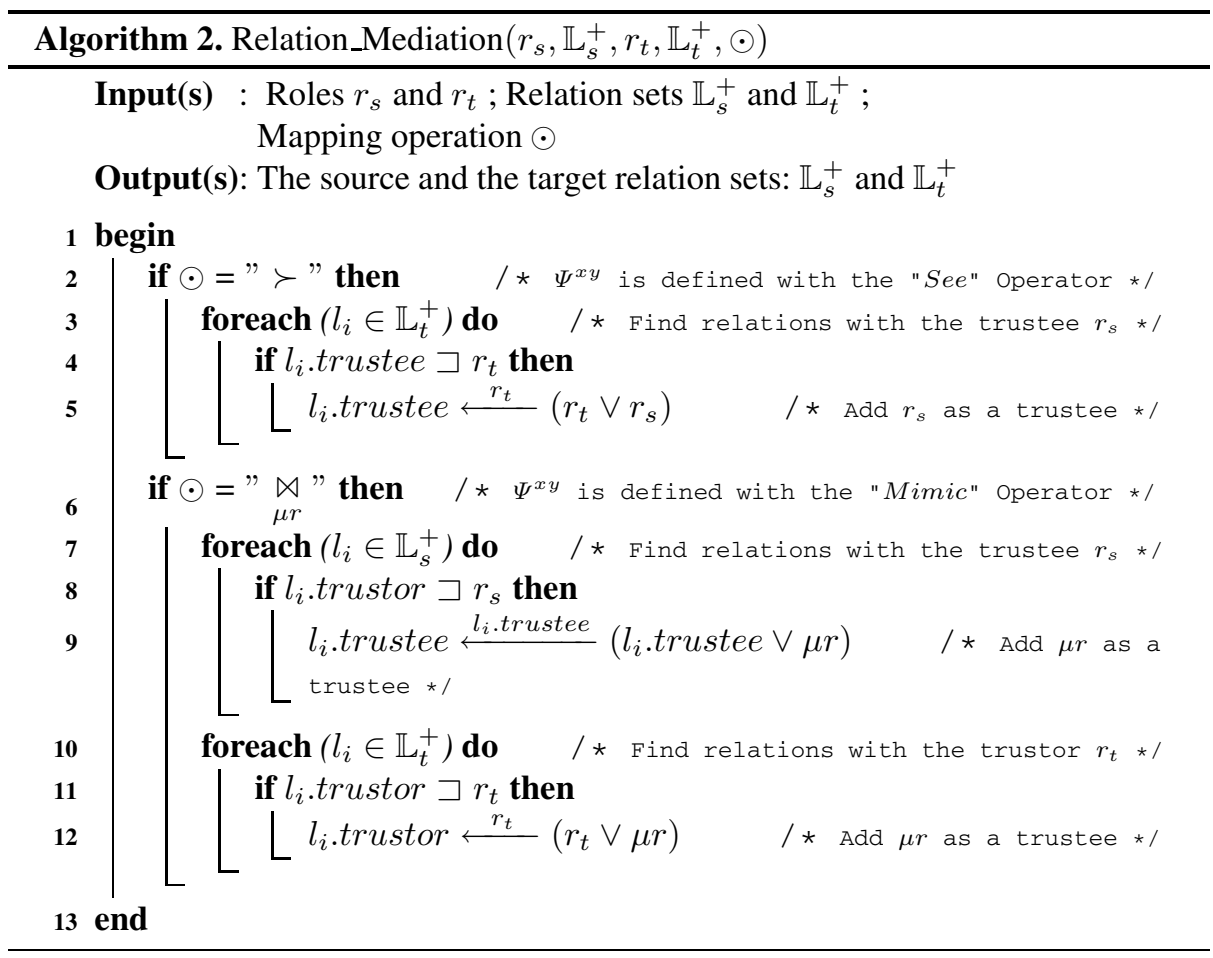




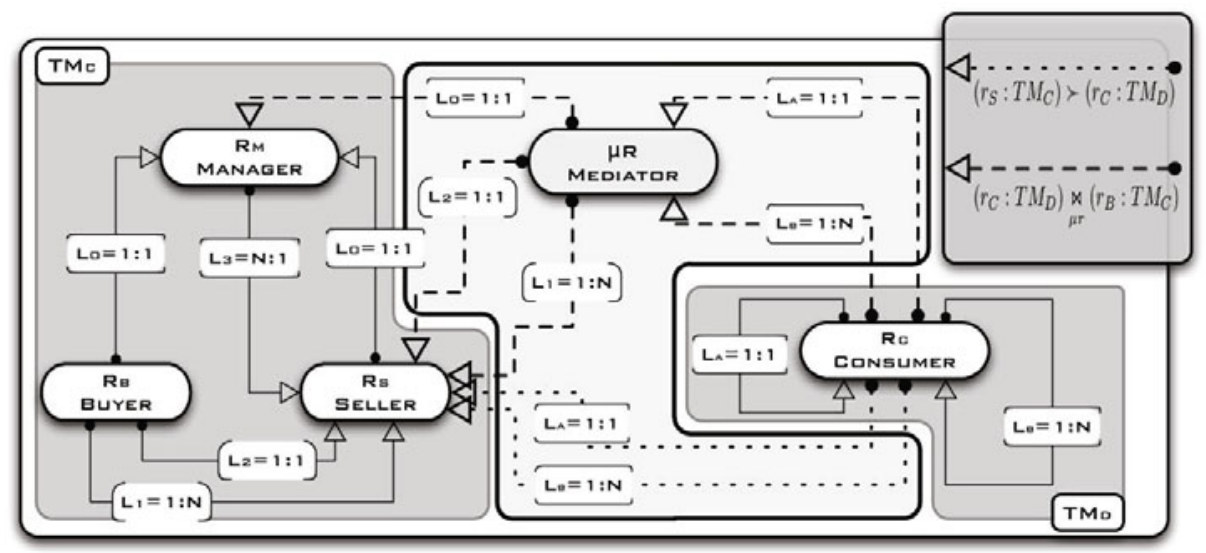

Fig. 3. Trust graph $T G_{C D}$

operator. The See operator defines which local trustee (target role $r_{t}$ ) corresponds to the source role $\left(r_{s}\right)$. Therefore, all the relations $l_{i}$ (from the source trust model) that consider the source role as a trustee $\left(l_{i}\right.$.trustee $\left.\sqsupset r_{t}\right)$ are extended with the target role (see lines 2-5). The Mimic operator introduces a new mediator role that plays trustees of the source role as a trustee in the source trust model, and plays the target role as a trustor in the target trust model. This leads to the corresponding extension of the trust models relations of $\mathbb{L}_{x}$ (see lines 7-9) and $\mathbb{L}_{y}$ (see lines 10-12).

Figure 3 depicts the trust graph $T G_{C D}$ resulting from the composition of $T M_{C}$ and $T M_{D}$, while Table 4 details the associated trust roles, metric and relations where new mediator role and extended relations are highlighted in grey. The composition relies on two mapping rules that allow a Customer of $T M_{D}$ to assess a seller of $T M_{C}$. The rule using the See operator represents how sellers are perceived in $T M_{D}$, while the second rule using the Mimic operator introduces a mediator role that enables Costumers to request $T M_{C}$ as Buyers. Thus, " $r_{B}: T M_{C} \succ r_{C}: T M_{D}$ " leads to extend the trustee element of $l_{a}$ and $l_{b}$ by replacing $r_{C}$ with $\left(r_{C} \vee r_{B}\right)$. The mapping rule " $r_{C}: T M_{D} \underset{\mu r}{\bowtie}$ $r_{S}: T M_{C}$ " extends the relations that sink into the role Customer (i.e., $l_{a}$ and $l_{b}$ ) with the mediator role $\mu \mathrm{r}$. In addition, all the relations that originate from the role Buyer (i.e., $l_{0}, l_{1}$ and $l_{2}$ ) also originate from the mediator role $\mu r$.

\subsection{Operation Mediation}

Operation mediation serves translating request operations from one model into requests in the other model, according to the mappings between roles defined using the Mimic operator. More precisely, consider a request operation by $r_{s}$ for a relation:

$<$ name="l", ctx="c", type="t", trustor=" $r_{s}$ ", trustee="tee", metric="v" $>$ of $T M_{s}$ where $l \in \mathbb{L}_{s}$, tor $\in \mathbb{R}_{s}$, while tee $\in \mathbb{R}_{t}$ and $r_{s}: T M_{s} \underset{\mu r}{\bowtie} r_{t}: T M_{t}$. Then, operation mediation first identifies the matching relations: 
Table 4. $T M_{C}$ and $T M_{D}$ Composition: Role, Metric, and Relation sets

\begin{tabular}{|c|c|}
\hline & les set $\mathbb{R}$ \\
\hline $\begin{array}{l}r_{S} \\
r_{B} \\
r_{M} \\
r_{C} \\
\mu r\end{array}$ & $\begin{array}{l}=<\text { name="Buyer" }> \\
=<\text { name="Seller" }> \\
=<\text { name="Manager" }> \\
=<\text { name="Customer" }> \\
=<\text { name="Customer Mediator" }>\end{array}$ \\
\hline Metr & ric set $\mathbb{M}$ \\
\hline & $\begin{array}{l}=<\text { name="Reputation", type="Probability" }> \\
=<\text { name="Recommendation", type="Probability" }> \\
=<\text { name="Rate", type="Five Semantic labels" }> \\
=<\text { name="Recommendation", type="Probability" }>\end{array}$ \\
\hline$\overline{\text { Rela }}$ & ation set $\mathbb{L}$ \\
\hline & 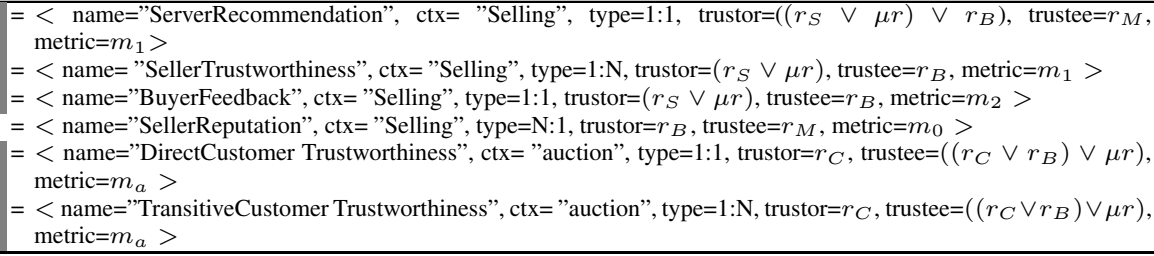 \\
\hline
\end{tabular}

$<$ name: string, ctx="c", type: string, trustor=" $r_{t}$ ", trustee="tee", metric: $\mathrm{m}>$ of $T M_{t}$

that should be requested in the target model using a request operation of $\mathbb{O}_{t}$. Replies are finally normalized using the mediation operation given by $\mu \mathbb{O}_{x y}$ for use in the source trust model. Operation mediation is practically implemented in a transparent way by the mediator that intercepts and then translates $r_{s}$ requests, as given in Algorithm 3. In the algorithm, the mediator interacts with $r_{s}$ (see lines 2-4) and $r_{t}$ (see lines 5-7). Then, the mediator computes the matching relation for each output relation (see lines 11-18) of the reply, where we assume that there is only one such relation (see lines 12-13) and requests its value using the appropriate request operation (see lines 16-18). We further consider that the mediator $(\mu r)$ embeds a library of mediation functions that translate and normalize heterogeneous trust metrics, which are invoked by mediation operations $\mu o$ (see lines 12-14). Finally, for each update (i.e., bootstrapping and refreshing) triggered by the response, as specified in the corresponding call element (see lines 19-20), the matching relations is sought in $\mathbb{L}_{t}$ (see line 23) and its value requested (see lines 25-28).

Figure 4 depicts the basic mediation process (left hand side) and its extension with update (right hand side), as performed by the mediator. First, the mediator receives the request in (step 1). Then, it invokes the corresponding request in the target model (steps 2 to 4 ) and upon receipt of the result, it normalizes the value using the mediation operation $\mu \mathbb{O}_{t s}$ (steps 5-6). Finally, the reply out is returned. In the case of update (on the figure right hand side), the relation matching the one given as input is sought in the target model using the mediation operation $\mu \mathbb{O}_{s t}$ (step 2), leading to invoke the corresponding update operation of the target model (step 3).

As an example, Table 5 gives the operation set $\mathbb{O}_{1,2}$ resulting from the composition of $T M_{C}$ and $T M_{D}$.

The response operation $o_{e}$ should be able to assess Sellers of $T M_{C}$ since its outputs (i.e., $l_{a}$ and $l_{b}$ ) contain relations that sink into the Seller role (see Table 4). To do so, $o_{e}$ is extended (see lines 9-18) to enable the mediator role $\mu r$ (when it performs this 


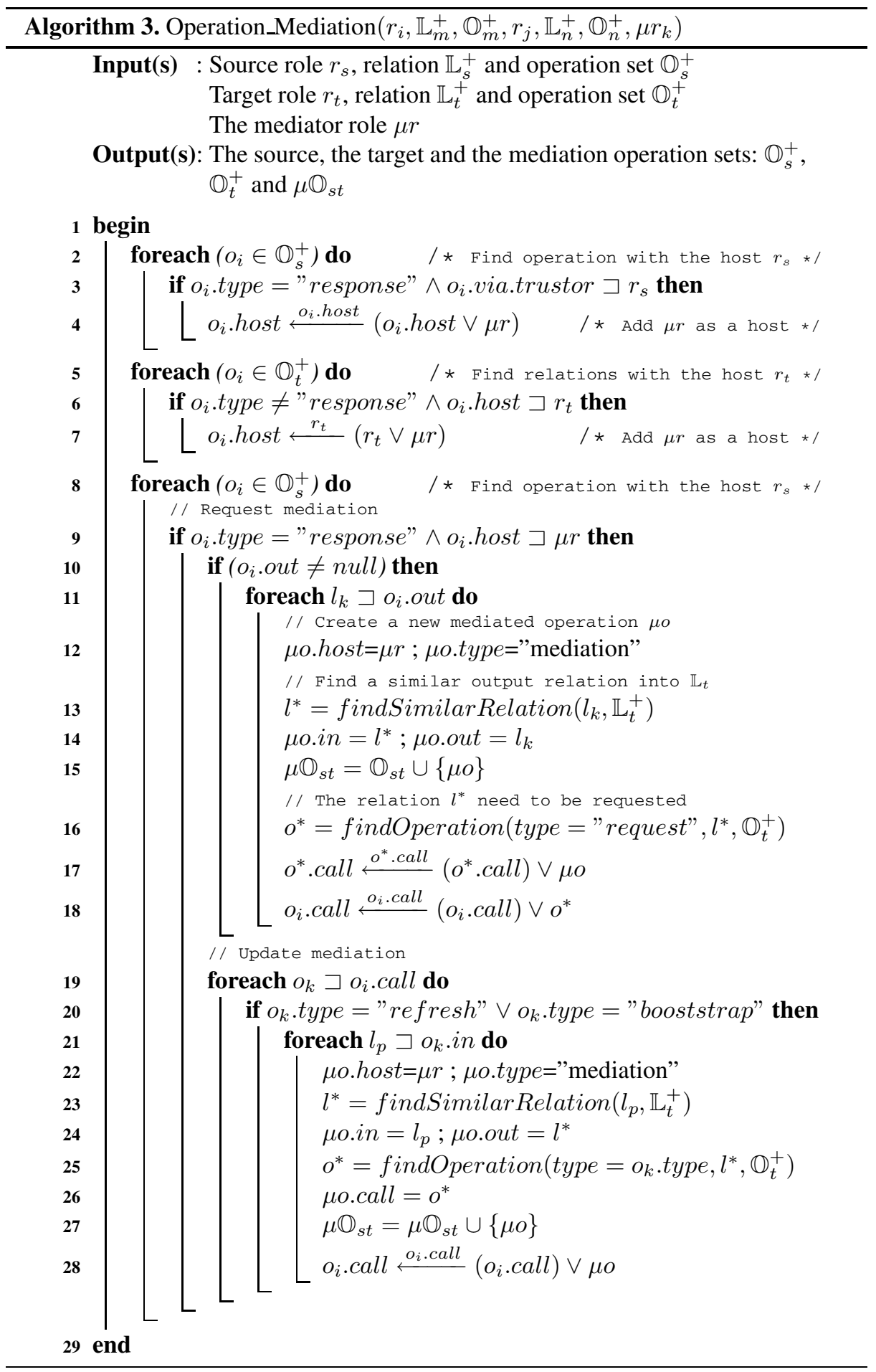




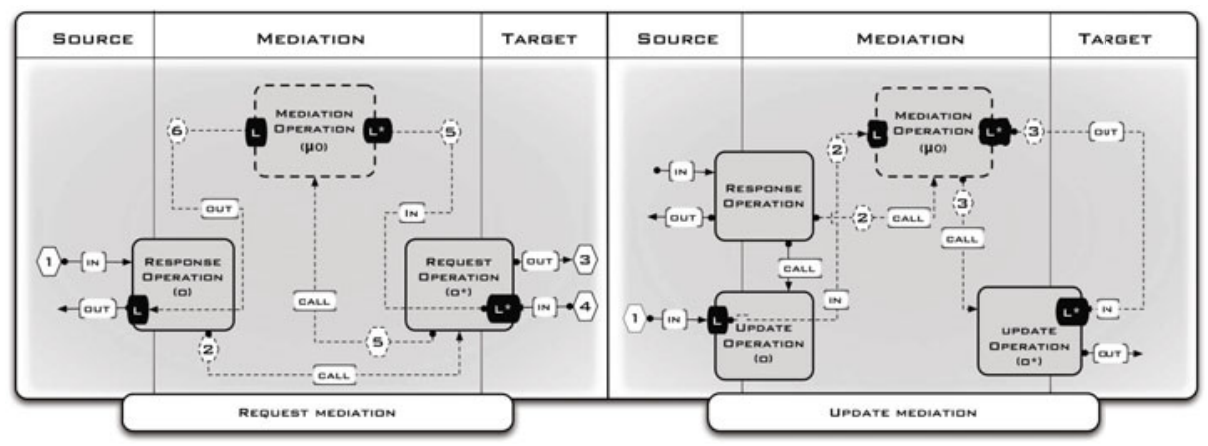

Fig. 4. Operation mediation process

Table 5. $T M_{C}$ and $T M_{D}$ Composition: Operation set

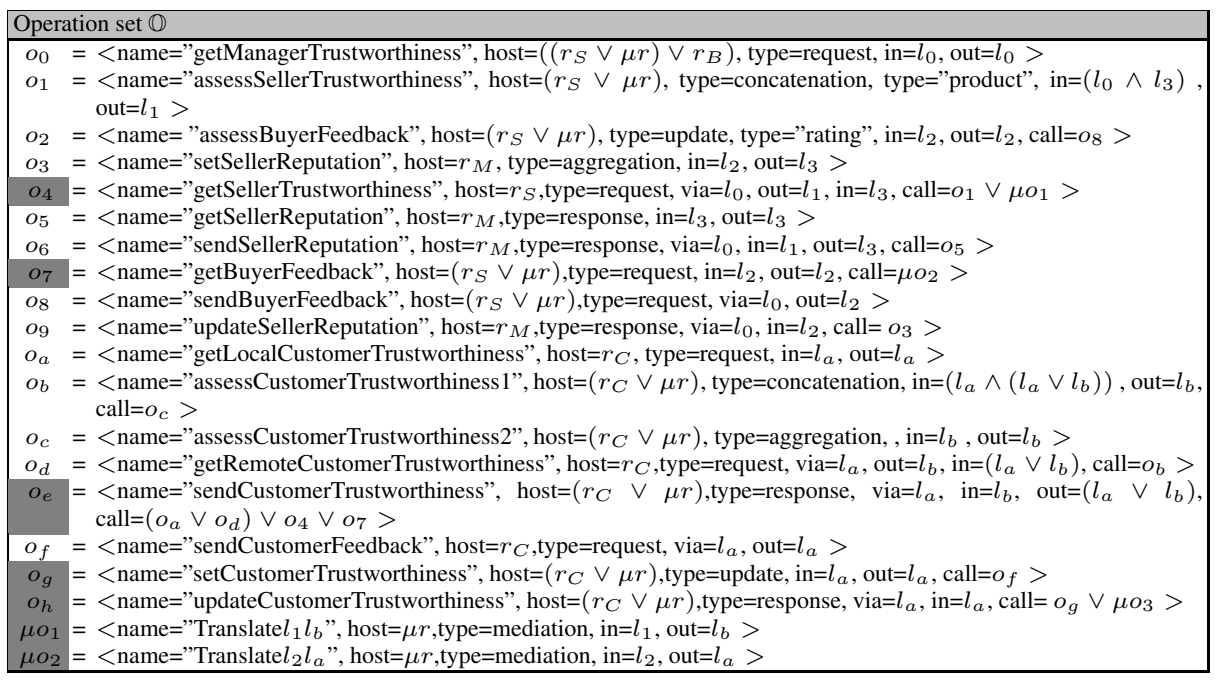

operation) to retrieve similar $o_{e}$ output relations in $T M_{C}$, i.e., the relations $l_{a}$ and $l_{b}$ that are respectively similar to $l_{1}$ and $l_{2}$. The operation $o_{e}$ can hence call $o_{4}$ or $o_{7}$ to search for $l_{1}$ or $l_{2}$. Then, as for $o_{e}$, the called operations are extended as well, by calling the mediation operations $\mu o_{1}$ and $\mu o_{2}$ to translate respectively $l_{1}$ and $l_{2}$ into $l_{b}$ and $l_{a}$. Thus, $o_{e}$ is able to reply the appropriate trust relationships which are interpretable by Customers. Moreover, Algorithm 3 (see lines 19-28) enables Customers feedback to be propagated to the Manager of the target model $T M_{C}$, so that the reputation of Sellers can be refreshed with the source model feedback. According to the resulting operation set (see Table 5), when the mediator role $\mu r$ performs the response operation $o_{h}$, it calls $\mu o_{3}$ to translate the feedback denoted by the relation $l_{a}$ into Buyer feedback, I.e., $l_{2}$. Then, $\mu o_{3}$ is able to call $o_{2}$ with the $l_{2}$ to advertise its feedback to $T M_{C}$ Manager. 


\section{Conclusion}

In this paper, we have introduced a trust meta-model as the basis to express and to compose a wide range of trust models. The composition of trust models enables assessing the trustworthiness of stakeholders across heterogeneous trust management systems. Such a composition is specified in terms of mapping rules between roles. Rules are then processed by a set of mediation algorithms to overcome the heterogeneity between the trust metrics, relations and operations associated with the composed trust models. We are currently implementing our approach as part of the Connect projec 3 where we have defined an XML-based description of the trust meta-model, which we call TMDL (i.e., Trust Model Description Language). Thus, mediators are synthesized on-the-fly given the TMDL description of Trust models.

As future work, we are also considering the implementation of a simulator to a priori assess the behavior of trust composition of given trust models and thus allows fine tuning of the mapping rules. We are also investigating the use of ontologies to specify the semantics of trust model elements and thus possibly infer the mapping rules as well as infer the similarity of trust relations from the semantics.

\section{References}

1. Abdul-Rahman, A., Hailes, S.: A distributed trust model. In: NSPW: New Security Paradigms Workshop, pp. 48-60. ACM Press, New York (1997)

2. Ahamed, S., Monjur, M., Islam, M.: CCTB: Context correlation for trust bootstrapping in pervasive environment. In: 2008 IET 4th International Conference on Intelligent Environments, pp. 1-8 (2008)

3. Ferraiolo, D.F., Sandhu, R., Gavrila, S., Kuhn, D.R., Chandramouli, R.: Proposed nist standard for role-based access control. ACM Transactions on Information and System Security 4(3), 224-274 (2001)

4. Fullam, K., Klos, T., Muller, G., Sabater, J., Schlosser, A., Topol, Z., Barber, K., Rosenschein, J., Vercouter, L., Voss, M.: A specification of the Agent Reputation and Trust (ART) testbed. In: Conference on Autonomous Agents and Multiagent Systems, pp. 512-518. ACM, New York (2005)

5. Grandison, T., Sloman, M.: A survey of trust in internet applications. IEEE Communications Surveys \& Tutorials 3(4), 2-16 (2009)

6. Haque, M., Ahamed, S.: An omnipresent formal trust model (FTM) for pervasive computing environment. In: 31st Annual International Computer Software and Applications Conference, COMPSAC 2007, vol. 1 (2007)

7. Jøsang, A., Pope, S.: Semantic constraints for trust transitivity. In: APCCM: 2nd Asia-Pacific Conference on Conceptual Modelling, pp. 59-68. Australian Computer Society, Inc., Newcastle (2005)

8. Jsang, A., Ismail, R.: The beta reputation system. In: Proceedings of the 15th Bled Electronic Commerce Conference, pp. 17-19 (2002)

9. Kaffille, S., Wirtz, G.: Engineering Autonomous Trust-Management Requirements for Software Agents: Requirements and Concepts. Innovations and Advances in Computer Sciences and Engineering, 483-489 (2010)

10. Kautz, H., Selman, B., Shah, M.: Referral Web: combining social networks and collaborative filtering. Communications of the ACM 40(3), 63-65 (1997)

\footnotetext{
${ }^{3}$ http://connect-forever.eu/
} 
11. Kim, Y., Doh, K.: Trust Type based Semantic Web Services Assessment and Selection. In: Proceedings of ICACT, IEEE Computer, pp. 2048-2053 (2008)

12. Marsh, S.: Formalising Trust as a Computational Concept. PhD thesis, University of Stirling, Scotland (1994)

13. Mui, L., Mohtashemi, M., Ang, C., Szolovits, P., Halberstadt, A.: Ratings in distributed systems: A bayesian approach. In: Proceedings of the Workshop on Information Technologies and Systems (WITS), pp. 1-7. Citeseer (2001)

14. Nurmi, P.: A bayesian framework for online reputation systems. In: International Conference on Internet and Web Applications and Services/Advanced International Conference on AICT-ICIW 2006, p. 121 (February 2006)

15. Paradesi, S., Doshi, P., Swaika, S.: Integrating Behavioral Trust in Web Service Compositions. In: Proceedings of the 2009 IEEE International Conference on Web Services, pp. 453-460. IEEE Computer Society, Los Alamitos (2009)

16. Quercia, D., Hailes, S., Capra, L.: TRULLO-local trust bootstrapping for ubiquitous devices. In: Proc. of IEEE Mobiquitous (2007)

17. Rahman, A., Hailes, S.: Supporting trust in virtual communities. In: IEEE Hawaii International Conference on System Sciences, p. 6007 (2000)

18. Saadi, R., Pierson, J.M., Brunie, L.: Establishing trust beliefs based on a uniform disposition to trust. In: ACM SAC: Trust, Reputation, Evidence and other Collaboration Know-how track. ACM Press, New York (2010)

19. Suryanarayana, G., Erenkrantz, J., Hendrickson, S., Taylor, R.: PACE: an architectural style for trust management in decentralized applications. In: Software Architecture, WICSA 2004, pp. 221-230. IEEE, Los Alamitos (2004)

20. Suryanarayana, G., Taylor, R.: SIFT: A Simulation Framework for Analyzing Decentralized Reputation-based Trust Models. Technical Report UCI-ISR-07-5 (2007)

21. Vercouter, L., Casare, S., Sichman, J., Brandao, A.: An experience on reputation models interoperability based on a functional ontology. In: Proceedings of 20th International Joint Conference on Artificial Intelligence, IJCAI 2007 (2007)

22. Wiederhold, G.: Mediators in the architecture of future information systems. Computer 25(3), 38-49 (2002)

23. Zhou, R., Hwang, K., Cai, M.: Gossiptrust for fast reputation aggregation in peer-to-peer networks. IEEE Transactions on Knowledge and Data Engineering, 1282-1295 (2008)

24. Zimmermann, P.R.: The official PGP user's guide. MIT Press, Cambridge (1995) 